\title{
Optical Code Division Multiple Access for a FTTH system
}

\author{
Bouregaa Mouweffeq ${ }^{1,2, *}$, Chikh-Bled Mohammed El Kebir ${ }^{1,3}$, Debbal Mohammed ${ }^{1,4}$, Ouadah Mohammed \\ Chamse Eddine ${ }^{1,3}$ and Chikh-Bled Hicham ${ }^{1,3}$ \\ ${ }^{I}$ Telecommunication Laboratory of Tlemcen (LTT), Tlemcen 13000, Algeria, \\ ${ }^{2}$ Mustapha Stambouli University, Mascara 29000, Algeria, \\ ${ }^{3}$ Abou-Bekr Belkaid University, Faculty of Technology, Tlemcen 13000, Algeria, \\ ${ }^{4}$ University Center Belhadj Bouchaib, Ain-Temouchent 46000, Algeria.
}

Received November 08, 2018; accepted December 21, 2018; published December 31, 2018

\begin{abstract}
Many multiple access techniques have been proposed and demonstrated to provide flexible solutions for FTTH network configurations. The performance of this system suffers because of the correlation properties that contribute to a high level of Multiple Access Interference (MAI), low system capacity (users), and lower transmission rate. In this paper, we have proposed Optical CDMA (OCDMA) as a configuration solution for FTTH networks to improve the performance of this type of network.
\end{abstract}

FTTH (Fiber To The Home) is a technology that brings optical fiber to the user, allowing access to the Internet and services associated with speeds of $10 \mathrm{Mbps}$ to $1 \mathrm{Gbps}$ symmetrical, much higher speeds than those accessible via the pair copper.

In this paper, we provide a detailed description of the FTTH system. We also explain how our OCDMA system works and provides promising solutions. We have also developed theoretical error probability expressions to study FTTH transmission system performance based on optical CDMA technique.

An optical line terminal, optical splitters and optical network terminal are the three components of the FTTH access network (Fig. 1) [1].

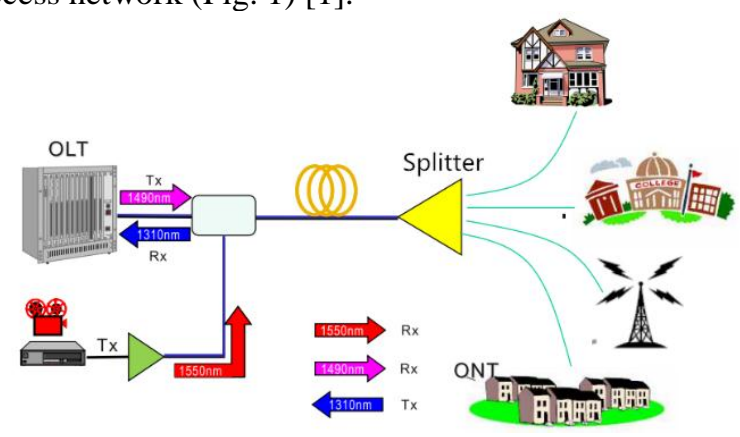

Fig. 1. FTTH network

An optical line terminal (OLT) (Fig. 2a) is the main element of the network and is usually placed in the local exchange. It is the engine that drives the FTTH system. Traffic scheduling, buffer control and bandwidth

\footnotetext{
*E-mail: bouregaa.abdou@yahoo.fr
}

allocation are the most important functions of the optical line terminal. Typically, an OLT operates using redundant DC power and has at least a 1 Line Card for the incoming Internet, a 1 System Card for on-board configuration [12].

An optical splitter (Fig. 2b) splits the power of the signal. That is to say, each fiber link entering the splitter may be split into a given number of fibers leaving the splitter. This enables sharing each fiber by many users. The passive optical splitter has the characteristics of a broad operating wavelength range, low insertion loss and uniformity, minimal dimensions, high reliability, and supporting network survivability and protection policy.

An ONT (Fig. 2c) is deployed at the customer premises. It is connected to the OLT by means of optical fiber and no active elements are present in the link. In the FTTH, the transceiver in the ONT is the physical connection between the customer premises and the central office OLT [3].

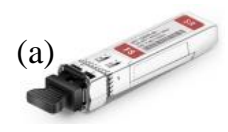

(b)
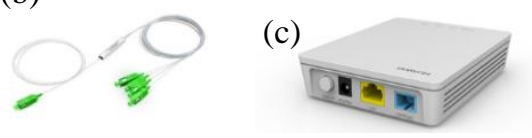

Fig. 2. (a) Optical Line Terminal (OLT); (b) Optical Splitter; (c) Optical Network Terminal (ONT).

In optical networks, the concept of CDMA or Optical Code Multiple Access Division (OCDMA) was introduced in the 1980s, with fibre optic communication systems enjoying a very high bandwidth. CDMA is based on the technique of a spread spectrum [4], which consists of distributing the power of a transmitted signal over a bandwidth much larger than that required for the transmission of information. Research on the OCDMA technique has developed in the context of broadband access networks. It is a case of considerable importance for operators, enabling them to offer many OCDMA services to several users. Many advantages are related to the use of CDMA for optical networks, such as:

- Users access the network simultaneously and asynchronously, 
- The transmitted information is secure; which is due to the encoding principle of data to be transmitted

- Several types of services can be transmitted simultaneously

- The system is scalable.

In an OCDMA system, spectral spread is achieved by directly multiplying the code sequences of users with their data to be transmitted. The coded data of users are coupled and sent simultaneously via the same fibre. On reception, the receiver multiplexes the received signal with a desired user sequence to extract the data sent as shown in Fig. 3 [5].

In optical communication systems using the CDMA technique, the characteristics of the codes are a determining factor for system implementation. The code must have the following characteristics:

- Each sequence must be easily distinguishable from all other sequences in the family (inter-correlation factor).

- Each sequence must be easily distinguishable from an offset version, and for all sequences of the family (autocorrelation factor).

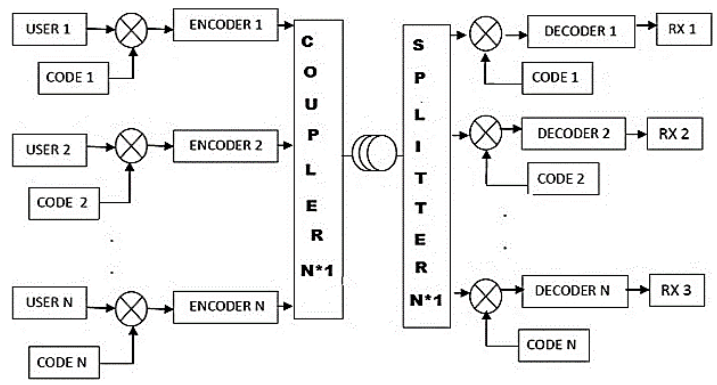

Fig. 3. OCDMA System.

Two categories of codes were studied, bipolar codes and unipolar codes. To overcome the phase control problem, unipolar sequences can be used. These are sequences that take values from the set $\{0,+1\}$, but we cannot have strict orthogonally with these codes. Codes that have been the focus are Orthogonal Optical Codes (OOC).

OOC is defined by the length of the sequence denoted by F (the number of chips), by W - the weight of the code indicating the number of chips of level " 1 " in a code sequence and the maximum number of users $\mathrm{N}$ that can be multiplexed using this family of sequences [6].

$$
N=\left\lfloor\frac{F-1}{W(W-1)}\right\rfloor,
$$

where \lfloor\rfloor is the operator which takes the integer portion of a lower value number. It should be noted that this theoretical expression gives an upper bound on the number of possible users in a family code.

The reception structure used at the end of the transmission chain is a very important element, whose function is to receive the signal transmitted in the optical fibre and then, from this signal, estimate the data transmitted by the desired user. Different structures of receipt may be used for an OCDMA-FTTH system [7]. In this work, we develop three structures of receiver: Conventional Correlation Receiver (CCR), CCR with Hard Limiter (HL+CCR) and Parallel Interference Cancellation (PIC).

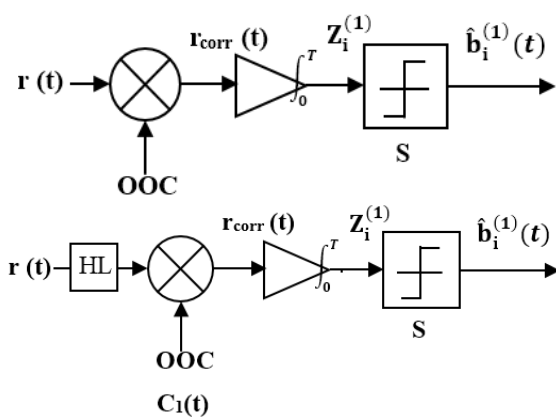

Fig. 4. (a) Conventional Correlation Receiver (CCR); (b) CCR WITH HARD LIMITER (HL-CCR)

The performance of an OCDMA-FTTH system can be limited by MAI, by the noise of optical components and by the impairments of an optical link. In order to study MAI cancellation, we consider all optical components to be ideal, i.e. errors are only due to MAI. In this case, we obtain error probability for the CCR [7]:

$$
P_{e C C R}=\frac{1}{2} \sum_{i=S}^{N-1} C_{N-1}^{i}\left(\frac{W^{2}}{2 F}\right)^{i}\left(1-\frac{W^{2}}{2 F}\right)^{N-1-i}
$$

$\mathrm{S}$ : the threshold of a receiver.

The HL-CCR reception structure presented in Fig.4 (b) uses the principle of the CCR; the only difference is an added function called Hard Limiter (HL). The hard limiter is an optical device that reduces received optical power. The error probability of the HL-CCR for the OCDMAFTTH system is written [7]:

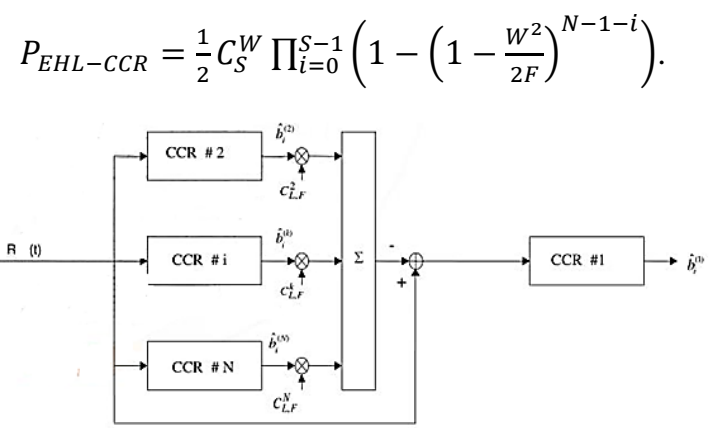

Fig. 5. Parallel Interference Cancellation Receiver (PIC)

The PIC receiver (Fig. 5) provides a simultaneous estimation of the contribution of each non-desired user. Then, an interference pattern is subtracted from the received signal before detecting, by means of a CCR, the 
data transmitted by the desired user. We have established in [7] the theoretical expression of error probability:

$$
\begin{gathered}
P_{E P I C}=\left(\frac{1}{2}\right)^{N} \sum_{N_{1}=S_{r}-1}^{N-1} \sum_{N_{2}=W+1-S_{F}}^{N-1-N_{1}} C_{N-1}^{N_{1}} \\
C_{N-1-N_{1}}^{N_{2}}\left(P_{I}\right)^{N_{2}}\left(1-P_{I}\right)^{N-1-N_{1}-N_{2}}, \\
\text { with } P_{I}=\frac{W^{2}}{F} \sum_{n_{1}=S_{r}-1}^{N_{1}} C_{N_{1}}^{n_{1}}\left(\frac{W^{2}}{F}\right)^{n_{1}}\left(1-\frac{W^{2}}{F}\right)^{N_{1}-n_{1}}
\end{gathered}
$$

We study the theoretical performance (BER: Bite Error Rate) of the OCDMA-FTTH system and we show the comparison between theoretical results obtained by numerical simulation for CCR, HL-CCR and PIC receivers.

We present some results as a function of the OOC parameters: the length $\mathrm{F}$, weight $\mathrm{W}$ and the number of users N. Our parameters set is: $F=361, W=4, N=30$. We chose these parameters because they are very close to the practice of installing FTTH networks

In Fig. 6a and Fig. 6b, we can see that the performance of 3 receivers improves when the length of the code increases (BER decreases). So, the higher the value of $F$ and $\mathrm{W}$, the less the desired user suffers from interference. Moreover, we can also note that the gain in performance provided by a PIC is even more important when F and W increase and better than a CCR and HL-CCR.

Figure $6 \mathrm{c}$ shows that the performance degrades (BER increases) when the number of users increases with each type of receiver. Therefore, the number of users increases, MAI increases and thus the performance of the system degrades more. Moreover, a PIC receiver is better performing than the other receivers.
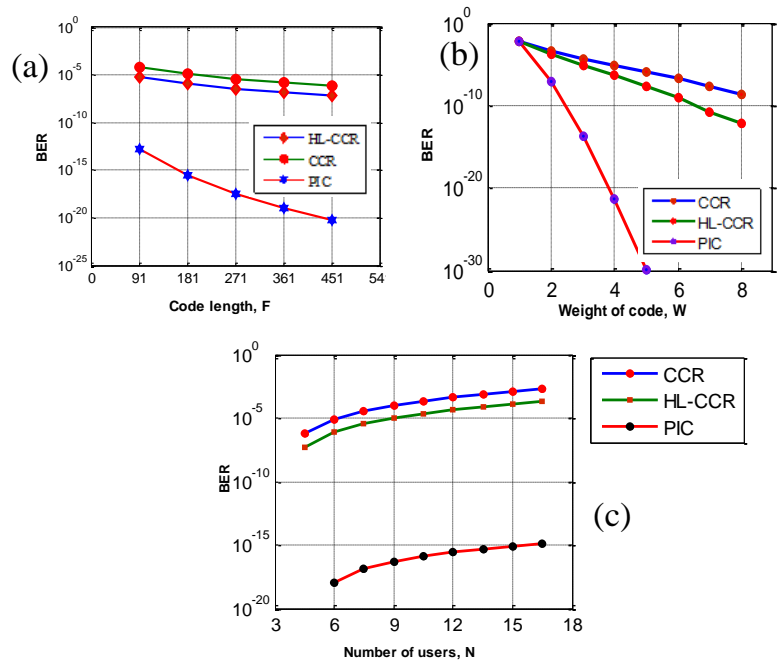

Fig. 6. (a) Receivers performance for $\mathrm{OOC}(\mathrm{F}, 4)$ with $\mathrm{N}=30$; (b) Receivers performance for OOC (361, W) with 30 users; (c) Receivers performance for OOC $(361,4)$ with $\mathrm{N}$ users.

We finish this study by comparing three types of receivers, made by calculating the minimal code to obtain the performance inferior to $10-9$ with a determined number of users $\mathrm{N}$. The results are reported in Table 1 .

The results grouped in the table show the optimal parameters of the code length $\mathrm{F}$ and the weight of code $\mathrm{W}$ and the transmission rate maximum in an FTTH network for numbers of users $\mathrm{N}$ multiplexed on the same optical fibre. We consider that the available electronic bandwidth (B) is $10 \mathrm{GHz}$, the decrease of the code length $\mathrm{F}$ permits to increase the data rate of each user because $\mathrm{D}=\mathrm{B} / \mathrm{F}$. All these results are obtained by several programs and simulations and are also compared with practical results and those published by domain researchers.

We can observe in Table I that we can reduce significantly the code length by using a PIC. Thus, if we consider that the available bandwidth (B) is $10 \mathrm{GHz}$, the maximum data rate for $\mathrm{N}=30$ users is $\mathrm{D}=\mathrm{B} / \mathrm{F}=5 \mathrm{Mbps}$ with a $\mathrm{CCR}$, and $\mathrm{D}=\mathrm{B} / \mathrm{F}=28 \mathrm{Mbps}$ with a PIC. Thus, the decrease of the code length $F$ permits to increase the data rate of each user.

Table I. Optimal Code Parameters (F, W) for BER $<10-9$

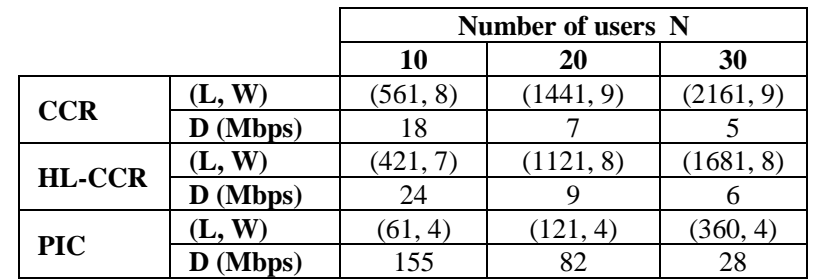

In conclusion, we have proposed a new FTTH configuration solution using the OCDMA technique that improves system capacity and data rate in integrating different services. Our OCDMA management solution easily scales the FTTH-PON system, supporting nearly a thousand customers using a single wavelength of monitoring. We have developed different coding and decoding parameters for our system.

\section{References}

[1] M. Mycek, M. Żotkiewicz, On cost of the uniformity in FTTH network design, Conf. on Transparent Opt. Networks 87 (2017).

[2] C.F. Lam, Passive Optical Networks- Principles and Practice, first ed. (British Library, USA 2007)

[3] M.K. Abdullah, W.T. P'ng, P.W. Lau, E.R. Tee, FTTH access network protection using a switch, Asia Pacific Conf. on Comm. (APCC) 3, 1219 (2003).

[4] J. Ronnakorn, S. Napat, L. Somkiat, Design and implement of GPONFTTH network for residential condominium, Conf. on Computer Science and Software Eng. 333 (2017).

[5] M. Bouregaa, M. Chikh-Bled, Intern. J. Hybrid Inform. Techn. 6225 (2013).

[6] M. Bouregaa, M. Chikh-Bled, Europ. Scien. J. 9, 322 (2013).

[7] M. Iwase, Y. Ishikawa, T. Komatsu, J. Kasahara, N. Hattori, M. Miura, N. Nakamura, K. Odaka, Furukawa Rev. 28, 8 (2005). 